OPEN ACCESS

Edited by:

Peng Qu,

National Institutes of Health (NIH),

United States

Reviewed by:

Hou Yuzhu,

University of Chicago, United States

Feng Geng,

The Ohio State University,

United States

*Correspondence:

Nan Zhang

zhangnan@jlu.edu.cn

Hong Xu

x_hong@jlu.edu.cn

${ }^{t}$ These authors have contributed equally to this work

Specialty section:

This article was submitted to

Cancer Immunity

and Immunotherapy,

a section of the journal

Frontiers in Immunology

Received: 22 December 2020

Accepted: 20 January 2021

Published: 02 March 2021

Citation:

Zhang L, Wang S, Wang Y, Zhao $W$, Zhang $Y$, Zhang $N$ and $X u H$ (2021) Effects of Hypoxia in Intestinal

Tumors on Immune Cell Behavior in the Tumor Microenvironment.

Front. Immunol. 12:645320. doi: 10.3389/fimmu.2021.645320

\section{Effects of Hypoxia in Intestinal Tumors on Immune Cell Behavior in the Tumor Microenvironment}

\author{
Luping Zhang ${ }^{1 \dagger}$, Shaokun Wang ${ }^{2 \dagger}$, Yachen Wang ${ }^{1}$, Weidan Zhao ${ }^{1}$, Yingli Zhang ${ }^{1}$, \\ Nan Zhang ${ }^{1 *}$ and Hong $X u^{1 *}$ \\ ${ }^{1}$ Department of Gastroenterology, The First Hospital of Jilin University, Changchun, China, ${ }^{2}$ Department of Emergency, The \\ First Hospital of Jilin University, Changchun, China
}

Background: Imbalanced nutritional supply and demand in the tumor microenvironment often leads to hypoxia. The subtle interaction between hypoxia and immune cell behavior plays an important role in tumor occurrence and development. However, the functional relationship between hypoxia and the tumor microenvironment remains unclear. Therefore, we aimed to investigate the effect of hypoxia on the intestinal tumor microenvironment.

Method: We extracted the names of hypoxia-related genes from the Gene Set Enrichment Analysis (GSEA) database and screened them for those associated with colorectal cancer prognosis, with the final list including $A L D O B, G P C 1, A L D O C$, and SLC2A3. Using the sum of the expression levels of these four genes, provided by The Cancer Genome Atlas (TCGA) and Gene Expression Omnibus (GEO) databases, and the expression coefficients, we developed a hypoxia risk score model. Using the median risk score value, we divided the patients in the two databases into high- and low-risk groups. GSEA was used to compare the enrichment differences between the two groups. We used the CIBERSORT computational method to analyze immune cell infiltration. Finally, the correlation between these five genes and hypoxia was analyzed.

Result: The prognosis of the two groups differed significantly, with a higher survival rate in the low-risk group than in the high-risk group. We found that the different risk groups were enriched by immune-related and inflammatory pathways. We identified activated MO macrophages in TCGA and GEO databases and found that CCL2/4/5, and CSF1 contributed toward the increased infiltration rate of this immune cell type. Finally, we observed a positive correlation between the five candidate genes' expression and the risk of hypoxia, with significant differences in the level of expression of each of these genes between patient risk groups.

Conclusion: Overall, our data suggest that hypoxia is associated with the prognosis and rate of immune cell infiltration in patients with colorectal cancer. This finding may improve immunotherapy for colorectal cancer.

Keywords: colorectal cancer, hypoxia, immune cell infiltration, tumor microenvironment, risk score 


\section{INTRODUCTION}

Since Stephen Paget proposed the "seed and soil" theory of cancer development and tumor metastasis (1), the understanding of the tumor microenvironment has gradually deepened. Several components of the tumor microenvironment contribute toward tumor occurrence and development (2, 3). An imbalance between nutrient supply and demand within the tumor often leads to hypoxia, glucose deficiency, and consequently an acidic tumor microenvironment (4). Specifically, tumor cells can use immune escape mechanisms to drive metastasis and invasion in anoxic environments (5). This has increased research interest into the relationship between hypoxia and the tumor immune microenvironment.

Hypoxia can reduce the activity of various immune cells in the tumor microenvironment and the production of corresponding immune stimulators to increase the release of suppressors and the expression of immune checkpoint inhibitors $(5,6)$, suggesting a close relationship between hypoxia and immune cells.

This study aimed to investigate the relationship between hypoxia-related genes and the immune microenvironment of colorectal cancer by (1) quantifying the influence of these genes on the tumor immune microenvironment, (2) screening hypoxiarelated genes associated with intestinal tumor prognosis, (3) developing a hypoxia risk score model, (4) validating the model's ability to predict patient prognosis and risk using data from The Cancer Genome Atlas (TCGA) and Gene Expression Omnibus (GEO) databases, (5) identifying the enrichment of model-related pathways, (6) using the hypoxia risk score as the entry point to explore differences in the infiltration rate of immune cells, and (7) identifying genes that affect immune cell infiltration to evaluate the relationship between hypoxia risk score and the tumor immune microenvironment.

\section{MATERIALS AND METHODS}

\section{Raw Data}

The intestinal cancer-related RNA-sequencing and clinical data used in this study were obtained from TCGA (https://portal.gdc. cancer.gov/) and GEO (gse39582) databases.

\section{Construction and Grouping of the Hypoxia Model}

The screening of hypoxia-related genes identified the genes independently related to the prognosis of intestinal cancer. We then multiplied the expression levels of each gene in TCGA and GEO databases by their respective expression coefficients, with the resulting sum defined as the risk score for each patient. Based on the median risk score value, the patients in the two databases were divided into high- and low-risk groups for the follow-up evaluations.

\section{Survival Analysis}

The survival and survminer $\mathrm{R}$ packages (The $\mathrm{R}$ Foundation for Statistical Computing, Vienna, Austria) were used to analyze the prognosis of 445 and 579 patients in TCGA and GEO databases, respectively. Patients in TCGA database were followed up for 12 years, while those in the GEO database were followed up for 16 years. The Kaplan-Meier method was used to plot the survival curves, and the log-rank test was used to evaluate the statistical significance between them, with $\mathrm{p}$-values $<0.05$ considered statistically significant.

\section{ROC Curve Analysis}

ROC curve analysis was conducted using the survival, survminer, and timeROC packages in R. The 1-, 3-, and 5-year survival rates of patients in TCGA and GEO databases were evaluated. The area under the ROC curve for the 1-, 3-, and 5-year survival rates increased gradually and exceeded 0.5 , which was defined as the threshold for the accurate prediction of survival by the model. Survival for each group is shown as risk columns and risk curves in Figures 2A, B.

\section{Heat Maps}

In this study, heat maps of gene expression were drawn using the pheatmap package in $\mathrm{R}$.

\section{PPI Network Analysis}

A network of anoxic genes was constructed using the STRING database. $\mathrm{R}$ software was used to select the 50 genes with the largest numbers of adjacent nodes for subsequent analysis.

\section{Cox Regression Analysis}

We applied the "survival" package in R and performed univariate Cox regression analysis to identify hypoxia-related genes that were closely related to prognosis. Univariate and multivariate prognostic analyses included factors such as age, sex, TNM stage, and the proposed risk score.

\section{Correlations Between Gene Expression and Hypoxia Risk}

After screening for genes that play a key role in immune cell regulation, the ggplot2, GGPUBR, and ggExtra packages in $\mathrm{R}$ were used to analyze the correlations between gene expression and the risk of hypoxia, and the differences in expression levels between patients at high- and low-risk of hypoxia.

\section{Gene Set Enrichment Analysis}

We downloaded the HALLMARK gene set and gene symbols from the GSEA website (https://www.gsea-msigdb.org/gsea/index.jsp) to extract hypoxia-related genes. The entire transcriptome of all tumor samples was used for GSEA, and only gene sets with nominal pvalues $<0.05$ and FDR q values $<0.06$ were considered significant.

\section{RESULTS}

\section{Extraction and Screening of Hypoxia- Related Genes}

We first downloaded the HALLMARK gene sets. The gene symbol set was obtained from the Gene Set Enrichment Analysis (GSEA) website (https://www.gsea-msigdb.org/gsea/index.jsp), which 
provides the names of all hypoxia-related genes. We then used the Search Tool for the Retrieval of Interacting Genes/Proteins (STRING) (http://string-db.org/cgi/input.pl), a protein-protein interaction (PPI) network database, to construct a model of PPIs between hypoxia-related genes (Figure 1A). By counting the number of adjacent nodes for each protein, we identified the core genes. The top 50 core genes with adjacent nodes are presented in Figure 1B. We then downloaded the intestinal tumor gene expression data and associated clinical information available from TCGA database. We extracted the data on hypoxiarelated gene expression and screened genes associated with prognosis using univariate Cox regression analysis (Figure 1C). Among the identified genes, $A L D O B$ was associated with a low risk, while five genes (GPC1, ALDOC, ENO2, SERPINE1, and $S L C 2 A 3)$ were associated with a high risk of developing tumor malignancy. The multivariate Cox regression analysis of genes associated with patient prognosis revealed four genes (Figure 1D) that were then used to construct prognostic models. These four genes ( $A L D O B, G P C 1, A L D O C$, and $S L C 2 A 3)$ had model coefficients of $-0.1574,0.2994,0.2647$, and 0.2074 , respectively.

\section{Effects of Hypoxia-Related Genes on Prognosis}

Multivariate Cox regression analysis identified four hypoxiarelated genes associated with intestinal tumor prognosis, which were used for modeling. We multiplied the expression levels of these genes (as reported in TCGA and GEO databases) by the corresponding coefficients to obtain the risk score for each patient. The median risk score value was then used to divide the patients in TCGA database into high- and low-risk groups. The subsequent survival analysis revealed significant differences between the high- and low-risk groups ( $\mathrm{p}<0.05$; Figures 2A, B). Receiver operating characteristic (ROC) curve analysis was used to verify the accuracy of the survival estimates derived from the present model, showing a gradually increasing accuracy of predicting the 1-, 3-, and 5-year survival rates of patients

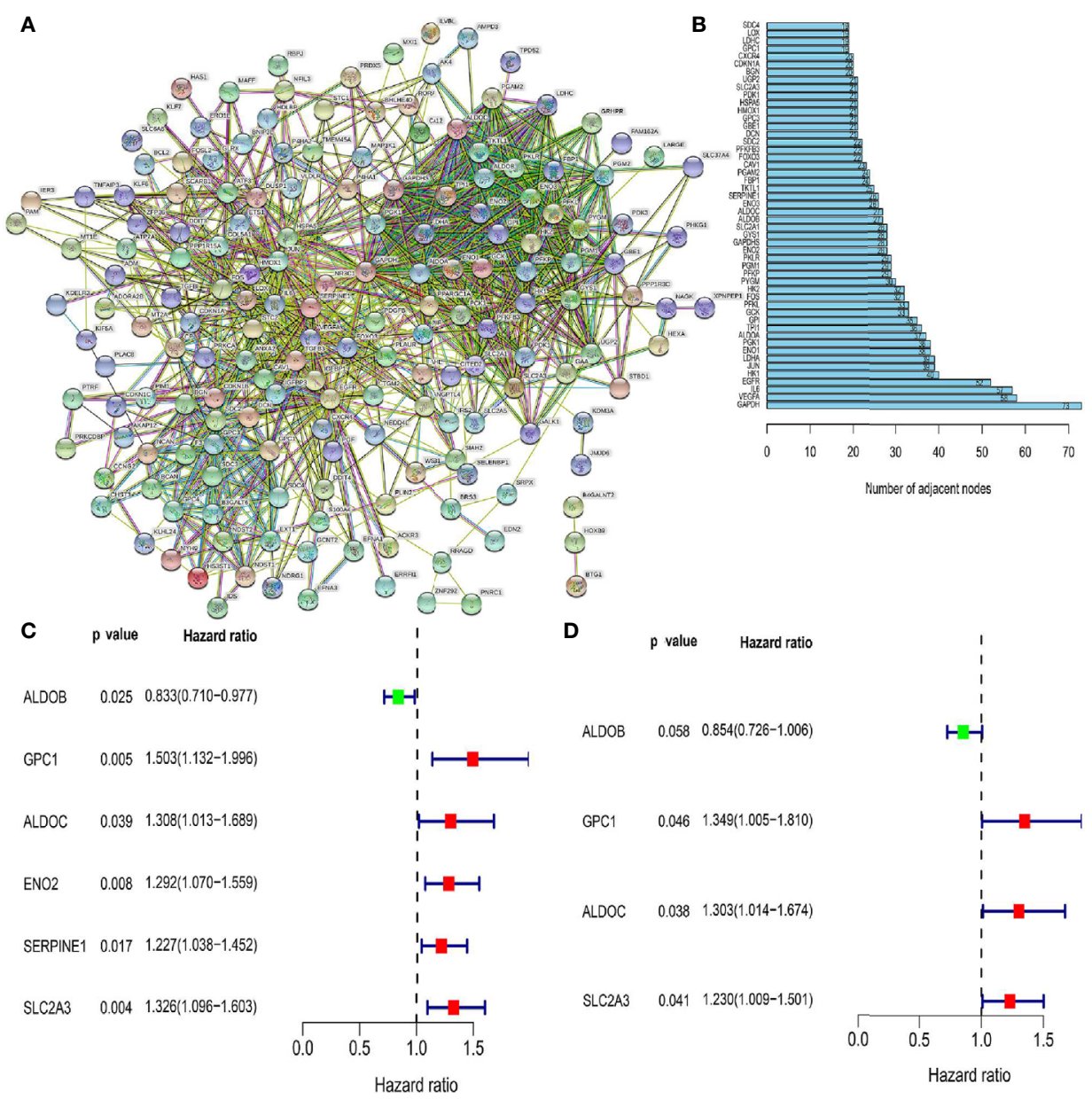

FIGURE 1 | Screening for hypoxia-related genes and their relationship with patient prognosis. (A) Protein-protein interaction network containing members with interaction confidence values $>0.4$. (B) The top 50 genes selected based on the number of nodes and their sub-nodes. (C) Univariate Cox regression analysis identified candidate genes with p-values $<0.05$. (D) Among the genes related to colorectal cancer prognosis, the genes shown are those independently related to patient prognosis in a multifactor prognostic model of hypoxia. 

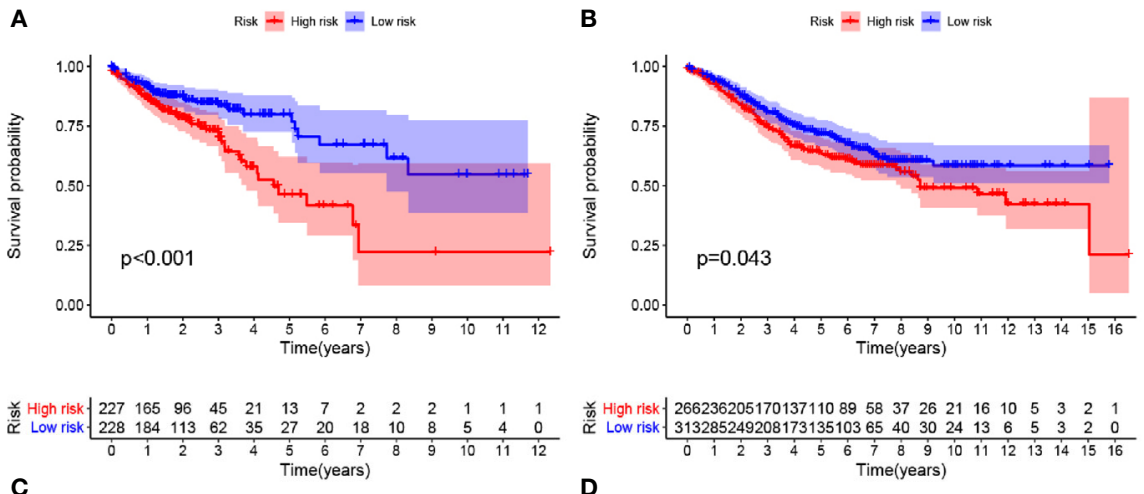

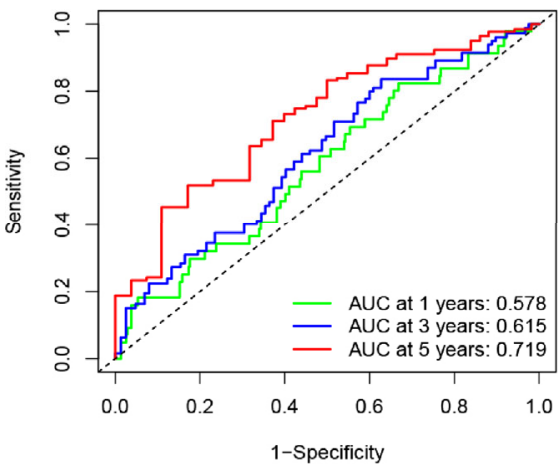

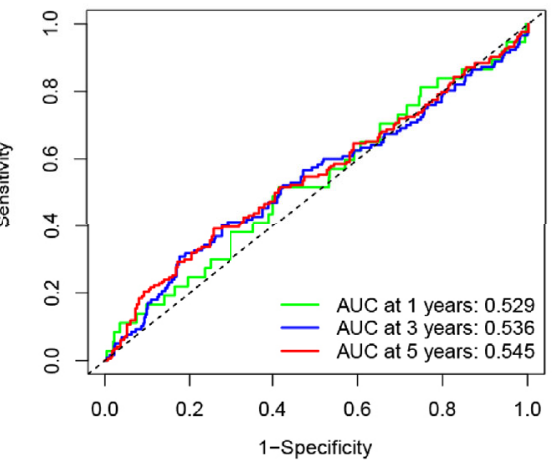

FIGURE 2 | Effects of the hypoxia model on patient prognosis. (A, B) Kaplan-Meier survival curves for patients with colon cancer in The Cancer Genome Atlas and Gene Expression Omnibus databases, stratified according to risk scores (high vs. low); comparisons of the median survival time in both groups with log-rank tests $(p<0.01$ and $p=0.043$, respectively). (C, D) Receiver operating characteristic curve analysis of the prognostic accuracy of the model.

included in TCGA database (Figure 2C). The area under the ROC curve for the GEO database was > 0.05 (Figure 2D), indicating that the model accurately predicted the survival rate. To value the survival rate of patients more intuitively in the highand low-risk groups, we used risk histograms to show the differences in survival status between the two databases (Figures 3A, B). We observed a higher proportion of surviving patients in the low-risk group than in the high-risk group. These results further demonstrated that our model effectively distinguished between high- and low-risk patients. We also analyzed the interactions between the hypoxia-related genes that were identified as affecting patient prognosis in the model (Figures 3C, D). We further demonstrated the relationship between patient risk and survival using risk curves, in which the risk scores for both groups of patients were plotted (Figures 3E, F). We observed a longer survival time in the low-risk group than in the high-risk group. Moreover, the number of deaths in the low-risk group decreased over time (Figures 3G, H). Finally, we compared the expression level of each gene included in the model between the high- and low-risk groups using thermography (Figures 3I, J).

\section{Effects of Different Clinical Characteristics on Intestinal Tumor Prognosis}

Clinical characteristics differ in the impact they have on patient prognosis. Thus, we analyzed the impact of clinical characteristics on the prognosis of patients included in TCGA and GEO databases. We first used univariate Cox regression analysis to evaluate the impact of clinical characteristics on the survival time and prognosis of patients included in the two databases (Figures 4A, B). We found that patient sex affected neither survival nor prognosis, while other factors affected survival and prognosis and were associated with increased risk. However, the p-value of the risk score in our model was $<0.05$ for patients in TCGA database, indicating that the risk score also affected patient prognosis and survival. In contrast, the p-value was $>0.05$ for patients in the GEO database. Multivariate analysis of these factors showed that the $\mathrm{p}$ values for age and tumor-node-metastasis (TNM) stage were both $<0.05$, indicating that these variables were independent prognostic factors (Figures 4C, D). We also observed differences in the expression levels of hypoxia-related genes between patients with different $\mathrm{T}$ stages included in the two databases (Figures $4 \mathbf{E}-\mathbf{H}$ ). The expression level of SLC2A3 in different T stages (T1, T2, T3, and T4) differed significantly between patients in TCGA and GEO databases $(\mathrm{p}<0.05)$.

\section{Enrichment of Pathways in Hypoxia- Related Risk Groups}

We observed differences in the enrichment level of hypoxiarelated genes and associated pathways between the high- and low-risk groups. To understand the level of pathway enrichment, we used GSEA software (UC San Diego, San Diego, CA, USA; Broad Institute, Cambridge, MA, USA) to compare the pathways 

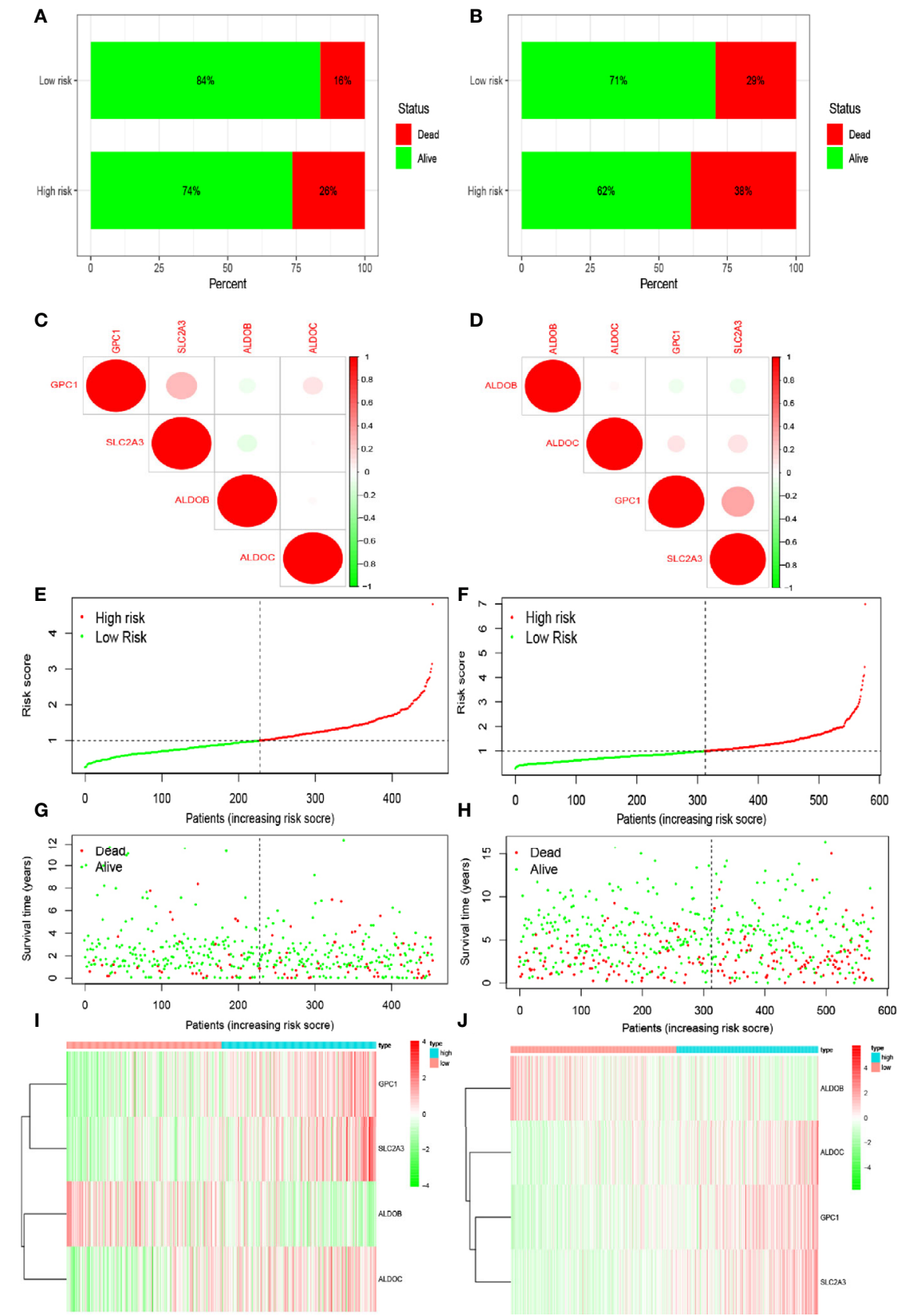

FIGURE 3 | Prediction of patient risk in the hypoxia model and the expression levels of the genes included in the model. (A, B) Patient survival in The Cancer Genome Atlas (TCGA) and Gene Expression Omnibus (GEO) databases. (C, D) Correlations between the genes included in the risk model based on TCGA and GEO databases. Positive and negative correlations are indicated in red and green, respectively. (E, F) Patient risk scores in TCGA and GEO databases. (G, H) Survival rates in the high- and low-risk patient groups in TCGA and GEO databases. (I, J) Heat maps of gene expression levels in the risk model for the high- and low-risk groups in TCGA and GEO databases.

between different risk groups. The high-risk group in TCGA database showed many enriched pathways related to apoptotic and immune functions (Figure 5A) compared with the low-risk group. Among these were apoptosis, hypoxia, interleukin (IL) 2/ signal transducer and activator of transcription (STAT) 5 signaling, IL6-STAT3 signaling, and the inflammatory response pathways. The other related pathways are shown in Table 1. In contrast, the pathways enriched in the low-risk group mainly included those associated with oxidative phosphorylation and lipid metabolism, among others. The pathways with false discovery rate (FDR) q values $>0.05$ are shown in Table 2 . The low-risk group of patients in the GEO database also showed the 

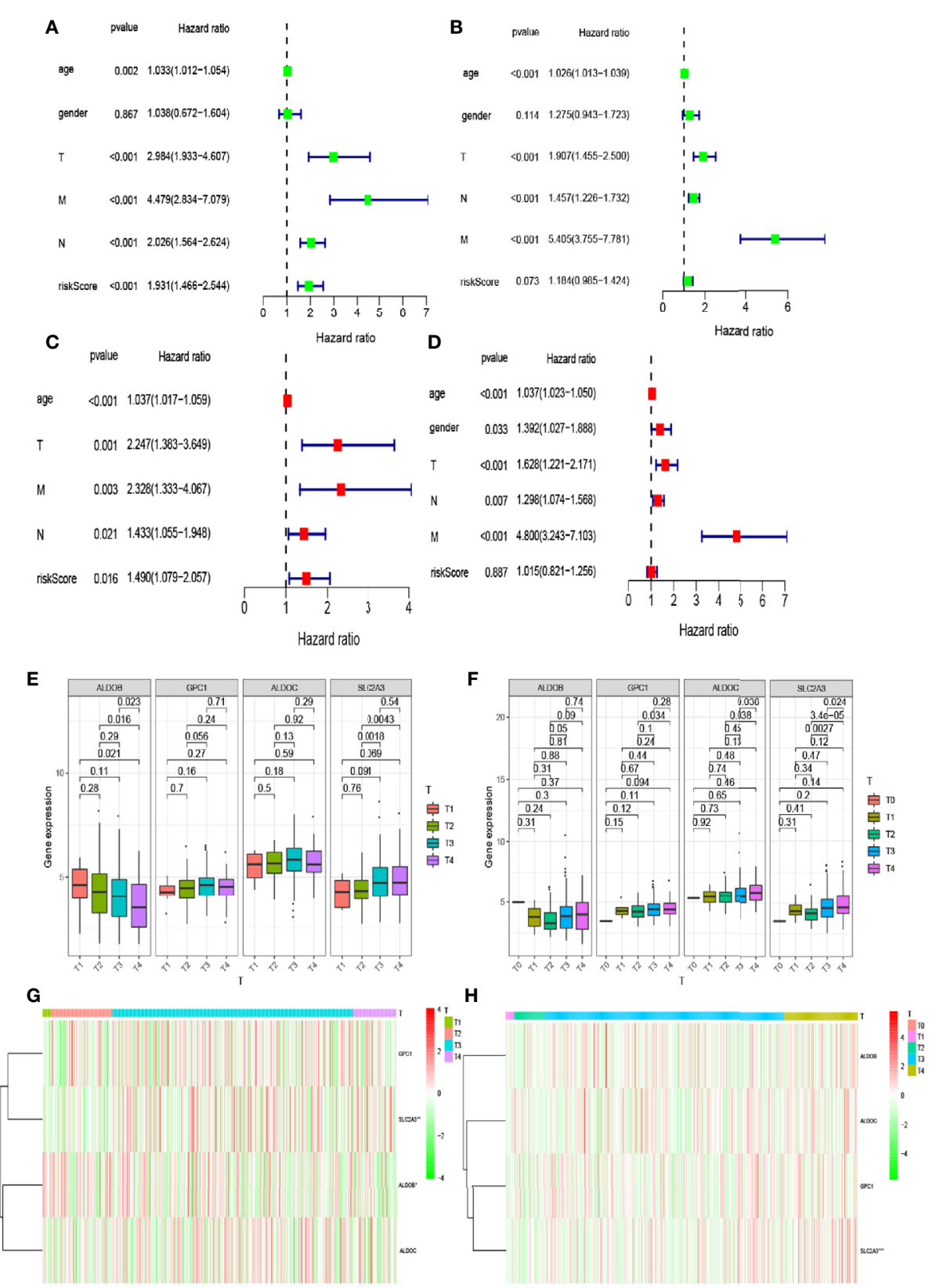

FIGURE 4 | Relationship between the risk model and clinical factors. (A, B) Single-factor prognostic analysis included age, sex, tumor-node-metastasis (TNM) stage, and the risk scores of patients with colorectal cancer in The Cancer Genome Atlas (TCGA) and Gene Expression Omnibus (GEO) databases. (C, D) Multifactor prognostic analysis included age, sex, TNM stage, and the risk scores of patients with colorectal cancer in TCGA and GEO databases. (E, F) Comparisons of the expression levels of various genes in the hypoxia model in TCGA and GEO databases for different T stages. (G, H) Heat maps showing the expression levels of genes in the risk model in TCGA and GEO databases for different T stages.

enrichment of apoptosis and IL2-STAT5 signaling pathways, in addition to, the p53 and peroxisome and phosphatidylinositol 3kinase-mammalian target of rapamycin serine/threonine protein kinase B signaling pathways (Figure 5B). The enrichment of other pathways is shown in Table 3 . The high-risk group also showed the enrichment of the Hedgehog and Wnt/beta-catenin signaling pathways. Pathways with FDR values $>0.05$ are shown in Table 4

\section{Immune Cell Infiltration}

The results of the pathway enrichment analysis of hypoxia-related genes for both risk groups in TCGA and GEO databases showed the 


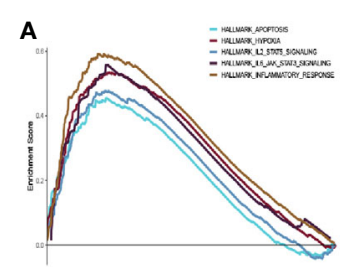

B
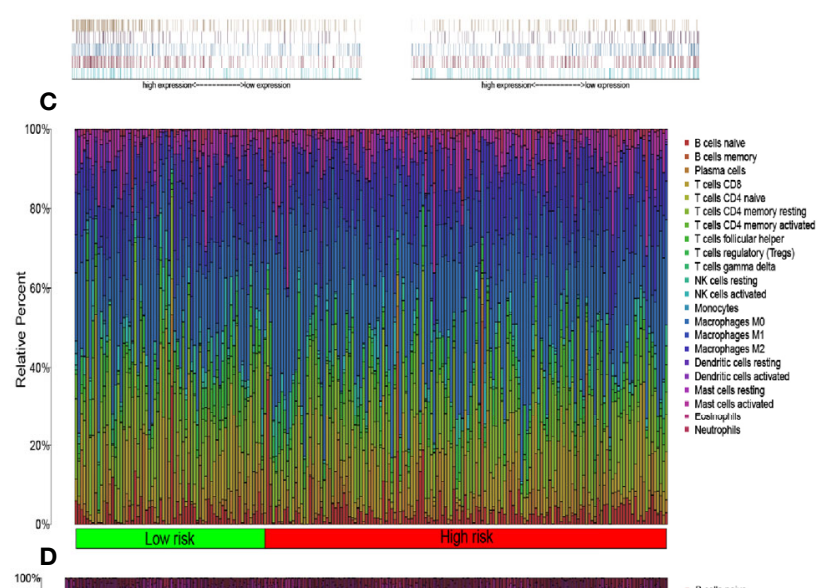

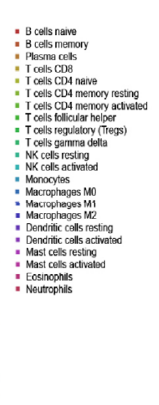

E

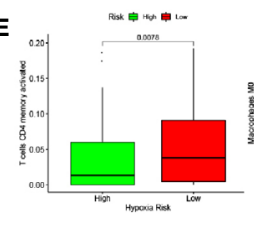

$\mathbf{F}$
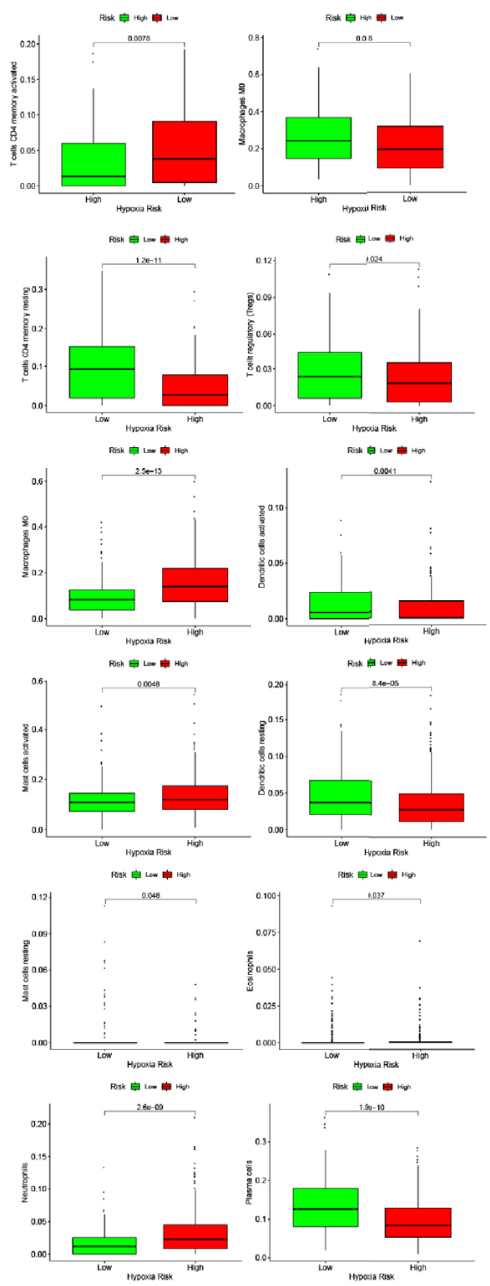

FIGURE 5 | Enrichment of hypoxia pathways and infiltration of hypoxia-related immune cells. (A) Enriched gene sets in the HALLMARK collection according to highrisk scores in The Cancer Genome Atlas (TCGA) database. Each line represents one particular gene set with a unique color, with upregulated genes appearing on the left side approaching the origin of the coordinates and downregulated genes appearing on the right side of the $\mathrm{x}$-axis. Only gene sets with nominal (NOM) $\mathrm{p}$ values $<0.05$ and false discovery rate (FDR) q values $<0.06$ were considered statistically significant. A selection of leading gene sets is shown in the plot. (B) The enriched gene sets in the HALLMARK collection by low-risk scores in the Gene Expression Omnibus (GEO) database. Only gene sets with NOM p-values <0.05 and FDR q values of $<0.06$ were considered statistically significant. A selection of leading gene sets is shown in the plot. (C, D) Heat map of hypoxia risk and immune cell infiltration in TCGA and GEO databases. (E) Immune cells whose infiltration is significantly associated with the risk of hypoxia in TCGA database ( $p<0.05$ ). (F) Immune cells whose infiltration is significantly associated with the risk of hypoxia in the GEO database $(p<0.05)$.

enrichment of pathways related to inflammation, immunity, and other factors. Based on these findings, we assessed the rate of immune cell infiltration in each risk group according to the constructed hypoxia-related gene model. Figures 5C, D shows the infiltration rate of immune cells in the high- and low-risk groups in TCGA and GEO databases, respectively. TCGA database showed differences in the infiltration rate of two types of immune cells in the high- and lowrisk groups (Figure 5E; $\mathrm{p}<0.05$ ). In contrast, the rate of infiltration of 10 types of immune cells differed between the high- and low-risk groups in the GEO database (Figure 5F; $\mathrm{p}<0.05$ ). Among them, the infiltration rate of activated M0 macrophages differed between the high- and low-risk groups in both databases. Using the Tracking Tumor Immunophenotype online platform (http://biocc.hrbmu.edu. $\mathrm{cn} / \mathrm{TIP} /$ index.jsp), we screened the immune-related genes for those that played important roles in the regulation of this immune cell type. Heat maps were then drawn to visualize the expression of these genes relative to that of hypoxia-related genes in the high- and low-risk groups of patients in TCGA and GEO databases (Figures 6A, B). The expression levels of CCL2/4/5, CSF1, and CX3CL1 were significantly different between the high- and low-risk groups in both databases $(\mathrm{p}<0.05)$. Next, we plotted the correlation curves between the expression levels of these four genes and the risk scores, which showed that the expression levels of these four genes were positively correlated with the patients' risk score. We also observed differences in the expression levels of these four genes between the high- and low-risk groups (Figures 6C-L). 
TABLE 1 | Pathway enrichment in the group of patients at high risk of hypoxia in The Cancer Genome Atlas database.

\begin{tabular}{|c|c|c|c|c|c|c|}
\hline NAME & SIZE & ES & NES & NOM p-val & FDR q-val & FWER p-val \\
\hline MYOGENESIS & 199 & 0.64095765 & 2.2988102 & 0 & 0 & 0 \\
\hline EPITHELIAL_MESENCHYMAL_TRANSITION & 198 & 0.7808052 & 2.2663856 & 0 & $9.73 \mathrm{E}-04$ & 0.001 \\
\hline COAGULATION & 138 & 0.5881612 & 2.2491221 & 0 & 6.49E-04 & 0.001 \\
\hline APICAL_JUNCTION & 200 & 0.5725273 & 2.210138 & 0 & 7.86E-04 & 0.002 \\
\hline HYPOXIA & 197 & 0.5341002 & 2.1750667 & 0 & 0.001029189 & 0.003 \\
\hline ANGIOGENESIS & 36 & 0.7042173 & 2.1259532 & 0 & 0.002047737 & 0.007 \\
\hline KRAS_SIGNALING_UP & 199 & 0.53099084 & 2.0836837 & 0 & 0.003663993 & 0.015 \\
\hline UV_RESPONSE_DN & 142 & 0.5923043 & 2.0315046 & 0.001988072 & 0.00553734 & 0.022 \\
\hline HEDGEHOG_SIGNALING & 36 & 0.6280092 & 2.0067165 & 0.003913894 & 0.005807516 & 0.026 \\
\hline RESPONSE & 200 & 0.5912703 & 2.0001185 & 0.003738318 & 0.005448199 & 0.027 \\
\hline COMPLEMENT & 200 & 0.5222255 & 1.9398772 & 0.001926782 & 0.008785588 & 0.045 \\
\hline IL2_STAT5_SIGNALING & 199 & 0.47638285 & 1.9347376 & 0.001923077 & 0.008227405 & 0.047 \\
\hline NOTCH_SIGNALING & 32 & 0.53026545 & 1.8844135 & 0.005928854 & 0.011401303 & 0.078 \\
\hline APICAL_SURFACE & 44 & 0.52808076 & 1.8692672 & 0 & 0.012468602 & 0.085 \\
\hline APOPTOSIS & 159 & 0.45459515 & 1.8656777 & 0.003846154 & 0.012308807 & 0.089 \\
\hline TNFA_SIGNALING_VIA_NFKB & 199 & 0.5475131 & 1.8406801 & 0.009380863 & 0.014613844 & 0.114 \\
\hline IL6_JAK_STAT3_SIGNALING & 87 & 0.5565036 & 1.7961316 & 0.0056926 & 0.020605113 & 0.158 \\
\hline TGF_BETA_SIGNALING & 54 & 0.47515002 & 1.6418401 & 0.034951456 & 0.059671924 & 0.381 \\
\hline ALLOGRAFT_REJECTION & 196 & 0.5172241 & 1.5929213 & 0.06 & 0.07578372 & 0.456 \\
\hline WNT_BETA_CATENIN_SIGNALING & 42 & 0.47698218 & 1.5757742 & 0.045454547 & 0.079787806 & 0.484 \\
\hline ESTROGEN_RESPONSE_EARLY & 198 & 0.32408136 & 1.4306225 & 0.055984557 & 0.15474646 & 0.701 \\
\hline CHOLESTEROL_HOMEOSTASIS & 73 & 0.38557705 & 1.4078543 & 0.10685484 & 0.16225821 & 0.734 \\
\hline KRAS_SIGNALING_DN & 199 & 0.34252158 & 1.3754886 & 0.07129095 & 0.17926803 & 0.779 \\
\hline UV_RESPONSE_UP & 156 & 0.28952506 & 1.2597458 & 0.12741312 & 0.27369708 & 0.891 \\
\hline INTERFERON_GAMMA_RESPONSE & 198 & 0.4217492 & 1.2542942 & 0.2848723 & 0.2676427 & 0.894 \\
\hline MITOTIC_SPINDLE & 198 & 0.31854162 & 1.188202 & 0.31697342 & 0.3293753 & 0.935 \\
\hline P53_PATHWAY & 195 & 0.27196786 & 1.1832048 & 0.21314742 & 0.3224601 & 0.937 \\
\hline ADIPOGENESIS & 198 & 0.27874216 & 1.1698754 & 0.27734375 & 0.32397935 & 0.943 \\
\hline GLYCOLYSIS & 199 & 0.26324964 & 1.1427033 & 0.26070037 & 0.34104726 & 0.956 \\
\hline ANDROGEN_RESPONSE & 99 & 0.30673286 & 1.1124011 & 0.33870968 & 0.36284208 & 0.966 \\
\hline ESTROGEN_RESPONSE_LATE & 198 & 0.2458808 & 1.1081452 & 0.2615694 & 0.35552585 & 0.967 \\
\hline HEME_METABOLISM & 193 & 0.23562211 & 1.0899739 & 0.30113637 & 0.36277255 & 0.972 \\
\hline MTORC1_SIGNALING & 197 & 0.24964637 & 0.90750676 & 0.5214724 & 0.5825153 & 0.997 \\
\hline PI3K_AKT_MTOR_SIGNALING & 105 & 0.209095 & 0.88849974 & 0.634 & 0.59227496 & 0.997 \\
\hline PANCREAS_BETA_CELLS & 40 & 0.23578233 & 0.70691955 & 0.8606061 & 0.83144087 & 1 \\
\hline INTERFERON_ALPHA_RESPONSE & 96 & 0.19630556 & 0.54184294 & 0.8392157 & 0.95013 & 1 \\
\hline
\end{tabular}

\section{DISCUSSION}

Hypoxia is a feature of tumor physiology specifically that of mechanisms associated with the acquisition of some malignant attributes, such as metastasis, invasion (7-9), and drug resistance (10). In these processes, hypoxia-related genes act on the corresponding pathways or on the regulating immune cells. Hypoxia-inducible factor (HIF) is activated during hypoxia. Some immunosuppressive factors, such as vascular endothelial growth factor, are HIF target genes, which affect both angiogenesis and immunosuppression (11). Hypoxia also results in upregulated EGFR expression, which promotes ligand-independent epidermal growth factor receptor signaling $(12,13)$. This process increases the rate of tumor glycolysis, resulting in metabolic competition (14). In our study, we screened for hypoxia-related genes in the gut and found that the core genes (ALDOB, GPC1, ALDOC, and SLC2A3) were closely related to patient prognosis. The rate of aldolase-B and fructose-bisphosphate B-driven fructose metabolism is significantly increased in patients with colon cancer and liver metastasis, and in those with colorectal villous polyps $(15,16)$. GPC1 has also been shown to be overexpressed in various malignancies $(17,18)$. A recent study has reported GPC1 enrichment in tumor-derived exosomes (17). In melanoma, NME1 has been shown to inhibit metastasis by activating ALDOC transcription (19). Solute carrier family 2, member 3 can increase glucose uptake in anoxic cells and, thus, increase the rate of glycolysis. These findings suggest that hypoxia-related gene expression levels are closely related to tumor development and metabolism (20); therefore, they were included in our hypoxia-related gene model.

With a deepening understanding of the mechanisms of hypoxia, its influence on tumor prognosis is also increasingly being understood $(21,22)$. To further investigate the relationship between the expression of hypoxia-related genes and patient prognosis, we evaluated patient prognosis by taking the product sum of the expression levels and the coefficients of $A L D O B$, GPC1, ALDOC, and SLC2A3 in TCGA and GEO databases as the risk score. The prognosis of patients in the high- and low-risk groups differed significantly; the survival rate of patients in the 
TABLE 2 | Pathway enrichment in the group of patients at low risk of hypoxia in The Cancer Genome Atlas database.

\begin{tabular}{|c|c|c|c|c|c|c|}
\hline NAME & SIZE & ES & NES & NOM p-val & FDR q-val & FWER p-val \\
\hline OXIDATIVE_PHOSPHORYLATION & 200 & -0.6354224 & -1.8517317 & 0.02 & 0.09670171 & 0.108 \\
\hline MYC_TARGETS_V1 & 196 & -0.55293024 & -1.5802475 & 0.091976516 & 0.29567894 & 0.452 \\
\hline E2F_TARGETS & 198 & -0.5320485 & -1.5347565 & 0.115830116 & 0.25026736 & 0.533 \\
\hline FATTY_ACID_METABOLISM & 156 & -0.37549332 & -1.483107 & 0.078125 & 0.24727894 & 0.605 \\
\hline PEROXISOME & 104 & -0.34722096 & -1.4177548 & 0.08267716 & 0.26794004 & 0.708 \\
\hline BILE_ACID_METABOLISM & 112 & -0.33064038 & -1.4166067 & 0.052529182 & 0.22491534 & 0.711 \\
\hline XENŌBIOTIC_METABOLISM & 197 & -0.23699556 & -1.0814732 & 0.31225297 & 0.6670434 & 0.973 \\
\hline MYC_TARGETS_V2 & 58 & -0.4065364 & -1.0632117 & 0.4333996 & 0.6178284 & 0.977 \\
\hline G2M_CHECKPOINT & 195 & -0.33763614 & -1.0606128 & 0.425 & 0.55348146 & 0.978 \\
\hline DNA_REPAIR & 150 & -0.28392524 & -1.0543846 & 0.39078155 & 0.50790316 & 0.981 \\
\hline PROTEIN_SECRETION & 96 & -0.30276886 & -1.0146313 & 0.45114344 & 0.5174334 & 0.986 \\
\hline REACTIVE_OXYGEN_SPECIES_PATHWAY & 49 & -0.27658382 & -0.960691 & 0.49802372 & 0.5441408 & 0.99 \\
\hline SPERMATOGGENESIS & 133 & -0.2188226 & -0.8435639 & 0.7213439 & 0.67120385 & 0.999 \\
\hline UNFOLDED_PROTEIN_RESPONSE & 110 & -0.18729709 & -0.7100693 & 0.8192771 & 0.8042398 & 1 \\
\hline
\end{tabular}

TABLE 3 | Pathway enrichment in the group of patients at high risk of hypoxia in the Gene Expression Omnibus database.

\begin{tabular}{|c|c|c|c|c|c|c|}
\hline NAME & SIZE & ES & NES & NOM p-val & FDR q-val & FWER p-val \\
\hline HEDGEHOG_SIGNALING & 35 & 0.47144 & 1.277345 & 0.14038461 & 1 & 0.749 \\
\hline WNT_BETA_CATENIN_SIGNALING & 41 & 0.430082 & 1.2600657 & 0.15187377 & 0.96750426 & 0.763 \\
\hline EPITHELIAL_MESENCHYMAL_TRANSITION & 195 & 0.5649699 & 1.176124 & 0.33539096 & 0.9005055 & 0.86 \\
\hline ANGIOGENESIS & 35 & 0.46240142 & 1.0144869 & 0.45738044 & 1 & 0.956 \\
\hline MYOGENESIS & 196 & 0.3335677 & 0.9412718 & 0.5278351 & 1 & 0.983 \\
\hline UV_RESPONSE_DN & 135 & 0.3387805 & 0.9249301 & 0.5495868 & 0.99989307 & 0.985 \\
\hline TGF_BETA_SIGNALING & 52 & 0.33623004 & 0.9151217 & 0.5373737 & 0.88053614 & 0.985 \\
\hline KRAS_SIGNALING_DN & 190 & 0.263007 & 0.8919948 & 0.642562 & 0.81584287 & 0.987 \\
\hline APICAL_SURFACE & 43 & 0.27583465 & 0.7981121 & 0.813278 & 0.91715556 & 0.996 \\
\hline KRAS_SIGNALING_UP & 192 & 0.30013293 & 0.7668628 & 0.806 & 0.8810992 & 0.997 \\
\hline ANDROGEN_RESPONSE & 93 & 0.22268894 & 0.7156268 & 0.86470586 & 0.8806738 & 0.998 \\
\hline COAGULATIONN & 136 & 0.2703017 & 0.67810416 & 0.9207317 & 0.8534861 & 0.998 \\
\hline
\end{tabular}

low-risk group was significantly higher than that of their counterparts. However, the ROC curve analysis, which aimed to evaluate the accuracy of the survival estimates, showed that the curves obtained from the GEO database corresponded poorly to the observed prognosis. Thus, we also analyzed the influence of other factors, including age, sex, TNM staging, and our proposed risk score, on patient prognosis. We concluded that our proposed risk score showed a good correspondence with patient prognosis. However, the results of the multivariate analysis in the GEO database were not statistically significant. This finding suggests that the risk score alone cannot be used as an independent prognostic factor. The samples in the GEO database were all colorectal adenocarcinoma samples that had been obtained in France. Because the tumor type and region are very specific, these samples may not accurately reflect the relationship between hypoxia and colon cancer prognosis. Moreover, the prognosis of colon cancer is related to disease stage and clinical, histological, genetic, and molecular factors, among others. These factors should be considered in future studies.

We next applied GSEA to identify pathways enriched in the high- and low-risk groups in the two databases. We found that most of the enriched pathways were related to inflammation, immune response, and apoptosis. Hypoxia and cell death in tumor tissue produce large amounts of cell debris and trigger the release of inflammatory factors, which can attract macrophages and monocytes, and can induce macrophage polarization. After polarization, macrophages secrete inflammatory factors (23). These findings suggest a close relationship between hypoxia, inflammation, and the immune response. In addition, some apoptosis-related genes, such as p53, which is a tumor suppressor gene, are closely related to tumor apoptosis. Nilay et al. (24) found that mutations in p53 in gastric and esophageal cancer cells can induce hypoxia signaling. This finding was confirmed in a study involving nude mice.

The results of many previous studies and those of the present study have shown that hypoxia can recruit immune cells into the tumor microenvironment. Hypoxia-induced tumor-derived cytokines, such as IL-10 and transforming growth factor-beta, can induce tumor-associated macrophages to differentiate into M2 macrophages with immunosuppressive effects (25). When monocytes are stimulated by inflammatory factors, such as interferon-gamma and lipopolysaccharide, they activate M1 macrophages, which can secrete inflammatory factors, such as IL-6 and tumor necrosis factor-alpha, and can phagocytize invasive pathogens and tumor cells $(23,26)$. Hypoxia can also 
TABLE 4 | Pathway enrichment in the group of patients at low risk of hypoxia in the Gene Expression Omnibus database.

\begin{tabular}{|c|c|c|c|c|c|c|}
\hline NAME & SIZE & ES & NES & NOM p-val & FDR q-val & FWER p-val \\
\hline OXIDATIVE_PHOSPHORYLATION & 180 & -0.5181038 & -1.8664485 & 0.017612524 & 0.052985493 & 0.035 \\
\hline FATTY_ACID_METABOLISM & 153 & -0.5680401 & -1.8461372 & 0.00203666 & 0.03559099 & 0.046 \\
\hline PEROXISOME & 101 & -0.53683174 & -1.8330911 & 0 & 0.029562779 & 0.051 \\
\hline CHOLESTEROL_HOMEOSTASIS & 71 & -0.54806143 & -1.6309602 & 0.021276595 & 0.15649396 & 0.239 \\
\hline ADIPOGENESIS & 189 & -0.41682148 & -1.5094645 & 0.035433073 & 0.32561186 & 0.449 \\
\hline XENOBIOTIC_METABOLISM & 193 & -0.52328324 & -1.5083747 & 0.028985508 & 0.2722546 & 0.449 \\
\hline REACTIVE_OXYGEN_SPECIES_PATHWAY & 47 & -0.4019267 & -1.376308 & 0.11977186 & 0.51835406 & 0.644 \\
\hline GLYCOLYSIS & 194 & -0.39022183 & -1.3331028 & 0.12045889 & 0.56736577 & 0.716 \\
\hline P53_PATHWAY & 188 & -0.3891394 & -1.2930824 & 0.124756336 & 0.61373806 & 0.757 \\
\hline BILE_ACID_METABOLISM & 109 & -0.45229995 & -1.2606792 & 0.17364016 & 0.63521326 & 0.791 \\
\hline ESTROGEN_RESPONSE_LATE & 197 & -0.3940817 & -1.2011873 & 0.156 & 0.73189086 & 0.858 \\
\hline UV_RESPONSE_UP & 153 & -0.34357396 & -1.175342 & 0.19662921 & 0.73222524 & 0.88 \\
\hline MYC_TARGETS_V2 & 56 & -0.4696598 & -1.1727189 & 0.37304688 & 0.6814103 & 0.88 \\
\hline MTORC1_SIGNALING & 189 & -0.38030684 & -1.1696959 & 0.29032257 & 0.6386857 & 0.882 \\
\hline PI3K_AKT_MTOR_SIGNALING & 103 & -0.29268706 & -1.132223 & 0.27868852 & 0.6835487 & 0.904 \\
\hline ESTROGEN_RESPONSE_EARLY & 193 & -0.32482004 & -1.0662084 & 0.33464566 & 0.79412293 & 0.936 \\
\hline PANCREAS_BETA_CELLS & 40 & -0.42545584 & -1.057426 & 0.38690478 & 0.767329 & 0.94 \\
\hline HEME_METABOLISM & 189 & -0.2667636 & -1.0508605 & 0.36575875 & 0.7411266 & 0.942 \\
\hline DNA_REPAIR & 144 & -0.298164 & -1.0362988 & 0.42330098 & 0.73253185 & 0.943 \\
\hline APOPTOSIS & 159 & -0.3165646 & -0.9227984 & 0.57114625 & 0.955858 & 0.981 \\
\hline SPERMATOGENESIS & 132 & -0.24104334 & -0.86871046 & 0.6871287 & 1 & 0.988 \\
\hline COMPLEMENT & 194 & -0.32908297 & -0.8492691 & 0.6442308 & 1 & 0.991 \\
\hline APICAL_JUNCTION & 188 & -0.27416155 & -0.83836824 & 0.64705884 & 1 & 0.992 \\
\hline MYC_TARGETS_V1 & 185 & -0.271399 & -0.80545324 & 0.628 & 1 & 0.995 \\
\hline INTERFERON_GAMMA_RESPONSE & 194 & -0.3529506 & -0.7729238 & 0.6927593 & 1 & 0.996 \\
\hline INTERFERON_ALPHA_RESPONSE & 92 & -0.36440378 & -0.7677859 & 0.6827853 & 1 & 0.996 \\
\hline ALLOGRAFT_REJECTION & 193 & -0.32352465 & -0.7621619 & 0.69921875 & 1 & 0.996 \\
\hline IL2_STAT5_SIGNALING & 193 & -0.24943894 & -0.74676377 & 0.8172888 & 1 & 0.997 \\
\hline UNFOLDED_PROTEIN_RESPONSE & 103 & -0.20758167 & -0.70057595 & 0.7745665 & 1 & 0.998 \\
\hline TNFA_SIGNALING_VIA_NFKB & 194 & -0.30261576 & -0.6995795 & 0.80670613 & 1 & 0.998 \\
\hline HYPOXIA & 190 & -0.24055389 & -0.6963746 & 0.8644401 & 1 & 0.998 \\
\hline MITOTIC_SPINDLE & 198 & -0.19739081 & -0.6422138 & 0.87279844 & 1 & 0.999 \\
\hline NOTCH_SIGNALING & 31 & -0.2139055 & -0.6397593 & 0.9166667 & 1 & 0.999 \\
\hline E2F_TARGETS & 190 & -0.2648517 & -0.622124 & 0.78019804 & 1 & 0.999 \\
\hline INFLAMMATORY_RESPONSE & 198 & -0.25689715 & -0.6020838 & 0.9265873 & 0.99692255 & 0.999 \\
\hline IL6_JAK_STAT3_SIGNALING & 87 & -0.23669887 & -0.56183934 & 0.9643564 & 0.99789304 & 1 \\
\hline PROTEIN_SECRETION & 95 & -0.16205812 & -0.5151241 & 0.97137016 & 0.9930653 & 1 \\
\hline G2M_CHECKPOINT & 185 & -0.19927993 & -0.5018363 & 0.89065605 & 0.9723166 & 1 \\
\hline
\end{tabular}

lead to immune escape through the role of immune cells (5); for example, hypoxia can reduce T-cell activity (27). Hypoxia is closely related to immune cell function. In our study, we observed significant differences in the level of activated M0 macrophages between the high- and low-risk groups in TCGA database. In the GEO database, the level of infiltration of activated CD4 memory T cells and M0 macrophages, activated and resting mast cells, and neutrophils differed significantly between the high- and low-risk groups. Both databases showed significant differences in the infiltration rate of activated M0 macrophages between the high- and low-risk groups. When we screened the genes that regulated this immune cell type, we found different levels of CCL2/4/5 and CSFlexpression between the high- and low-risk groups. Further analyses confirmed the significant correlation between the expression level of each of these genes and the risk of hypoxia.

Three of these four genes encode chemokines, which play a chemotactic role in immune cells, such as natural killer cells and monocytes, which are closely related to tumor development. CCL2 and CCL5 play important roles in prostate cancer metastasis and drug resistance $(28,29)$, while CCL4 is associated with the clinical characteristics of breast cancer (30). CSF1 is expressed in almost all tumors $(31,32)$. It recruits macrophages other than alveolar macrophages through CSF1 receptors and regulates their differentiation $(31,33)$. Macrophages, a major component of the tumor microenvironment, contribute to tumorigenesis by promoting angiogenesis, immunosuppression, invasion, and metastasis $(34,35)$.

In conclusion, hypoxia plays an important role in the tumor microenvironment. Screening for genes that can affect the rate of immune cell infiltration revealed a correlation between these genes and the hypoxia risk score. Our findings show that hypoxia-related genes can affect the prognosis of intestinal cancer and may play a role in immune infiltration in intestinal cancer. Analysis of the relationship between hypoxia and immune cells may improve immunotherapy and tumor treatment. 


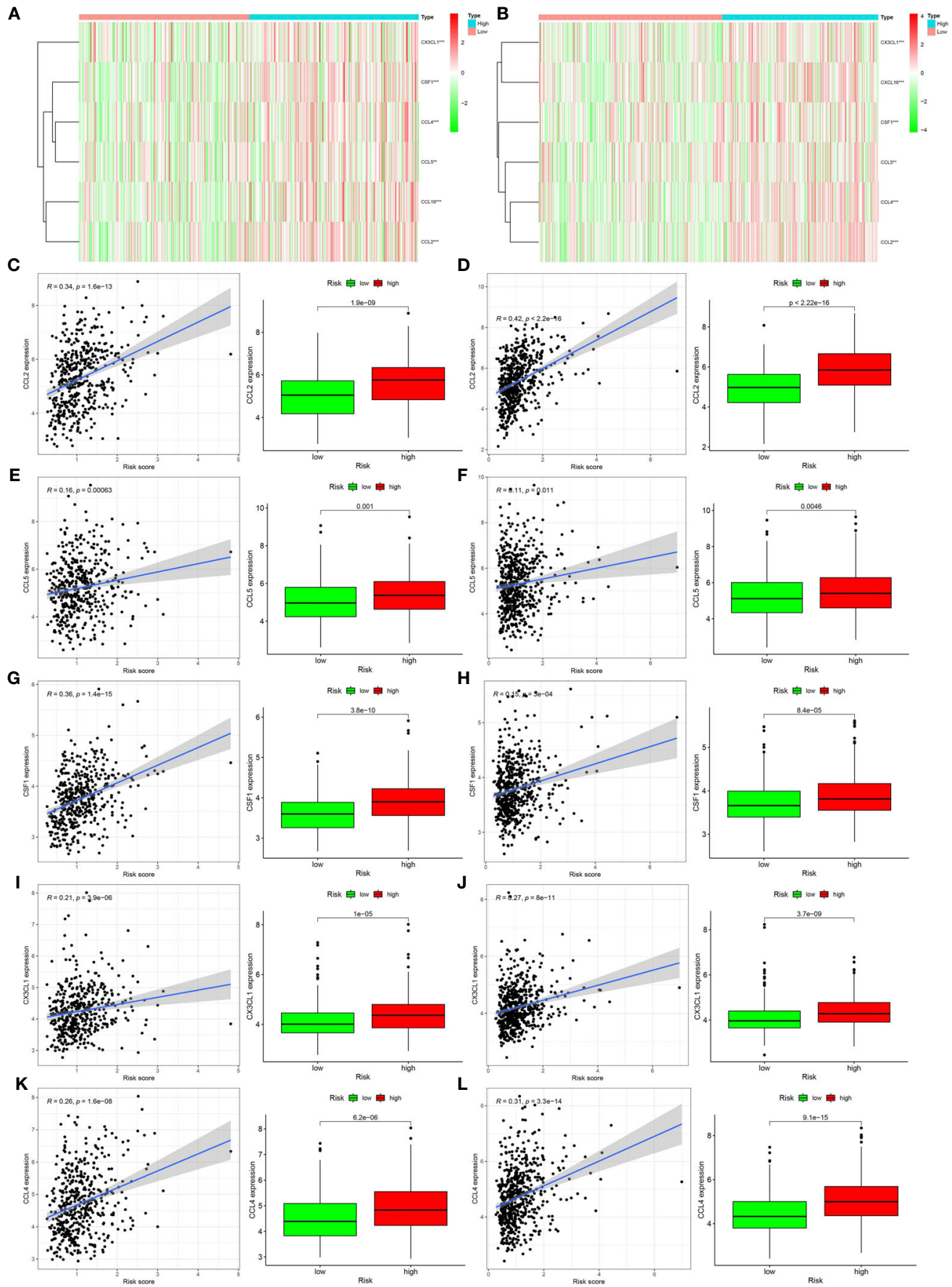

FIGURE 6 | Relationships between genes regulating immune cell behavior and hypoxia risk. (A, B) Heat maps showing the expression levels of genes regulating activated CD4 memory T cells and M0 macrophages in different hypoxia risk groups $\left({ }^{\star \star} \mathrm{p}<0.01 ;{ }^{* \star \star} \mathrm{p}<0.001\right)$. (C-L) Scatter plots showing the correlations of the expression of four genes from The Cancer Genome Atlas and Gene Expression Omnibus databases with immune cell regulation, showing differences in the expression levels between the different hypoxia risk groups $(p<0.05)$. The blue line in each plot is a fitted linear model indicating the relationship between gene expression and the risk of hypoxia. Pearson coefficients were used to assess the correlation between the two factors. The box plots show the differences in the levels of gene expression between groups at risk of hypoxia $(p<0.05)$. 


\section{DATA AVAILABILITY STATEMENT}

The original contributions presented in the study are included in the article/supplementary material. Further inquiries can be directed to the corresponding authors.

\section{ETHICS STATEMENT}

All participants provided a signed informed consent.

\section{AUTHOR CONTRIBUTIONS}

The data analysis and original writing of the draft were conducted by LZ and SW. HX and NZ came up with the design and critical revision of the manuscript. The original writing of the draft and its

\section{REFERENCES}

1. Stephen P. The distribution of secondary growths in cancer of the breast. Lancet (1889) 133(3421):571-3. doi: 10.1016/S0140-6736(00)49915-0

2. Hanahan D, Coussens Lisa M. Accessories to the Crime: Functions of Cells Recruited to the Tumor Microenvironment. Cancer Cell (2012) 21(3):309-22. doi: 10.1016/j.ccr.2012.02.022

3. Lebleu VS. Imaging the tumor microenvironment. Cancer J (2015) 21:174-8. doi: 10.1007/978-3-319-67577-0_15

4. Klemm F, Joyce JA. Microenvironmental regulation of therapeutic response in cancer. Trends Cell Biol (2015) 25(4):198-213. doi: 10.1016/j.tcb.2014.11.006

5. Barsoum IB, Koti M, Siemens DR, Graham CH. Mechanisms of hypoxiamediated immune escape in cancer. Cancer Res (2014) 74(24):7185-90. doi: 10.1158/0008-5472.CAN-14-2598

6. Multhoff G, Vaupel P. Hypoxia Compromises Anti-Cancer Immune Responses. Adv Exp Med Biol (2020) 1232:131-43. doi: 10.1007/978-3-03034461-0_18

7. Chaturvedi P, Gilkes DM, Wong CCL, Shitiz K, Luo W, Zhang H, et al. Hypoxia-inducible factor-dependent breast cancer-mesenchymal stem cell bidirectional signaling promotes metastasis. J Clin Invest (2013) 123(1):189205. doi: 10.1172/JCI64993

8. Cheng JC, Klausen C, Leung PCK. Hypoxia-inducible factor 1 alpha mediates epidermal growth factor-induced down-regulation of E-cadherin expression and cell invasion in human ovarian cancer cells. Cancer Lett (2013) 329 (2):197-206. doi: 10.1016/j.canlet.2012.10.029

9. Rankin EB, Giaccia AJ. Hypoxic control of metastasis. Science (2016) (6282):175-80. doi: 10.1126/science.aaf4405

10. Facciabene A, Peng X, Hagemann IS, Balint K, Barchetti A, Wang LP, et al. Tumour hypoxia promotes tolerance and angiogenesis via CCL28 and T(reg) cells. Nature (2011) 475(7355):226-30. doi: 10.1038/nature10169

11. Gavalas NG, Tsiatas M, Tsitsilonis O, Politi E, Ioannou K, Ziogas AC, et al. VEGF directly suppresses activation of $\mathrm{T}$ cells from ascites secondary to ovarian cancer via VEGF receptor type 2. Br J Cancer (2012) 107(11):1869-75. doi: 10.1038/bjc.2012.468

12. Franovic A, Gunaratnam L, Smith K, Robert I, Patten D, Lee S. Translational up-regulation of the EGFR by tumor hypoxia provides a nonmutational explanation for its overexpression in human cancer. Proc Natl Acad Sci (2007) 104(32):13092-7. doi: 10.1073/pnas.0702387104

13. Misra A, Pandey C, Sze SK, Thanabalu T. Hypoxia activated egfr signaling induces epithelial to mesenchymal transition (EMT). PloS One (2012) 7(11): e49766. doi: 10.1371/journal.pone.0049766

14. Wang Y, Roche O, Xu C, Moriyama EH, Heir P, Chung J, et al. Hypoxia promotes ligand-independent EGF receptor signaling via hypoxia-inducible factor-mediated upregulation of caveolin-1. Proc Natl Acad Sci (2012) 109 (13):4892-7. doi: 10.1073/pnas.1112129109 editing were by YW, WZ, and YZ. All authors contributed to the article and approved the submitted version.

\section{FUNDING}

Finance Department of Jilin (2018SCZWSZX-039); Finance Department of Jilin (JLSWSRCZX2020-083); Science and Technology of Jilin (20170623092TC-119).

\section{ACKNOWLEDGMENTS}

We would like to acknowledge the reviewers for their helpful comments on this study.

15. Lin WR, Chiang JM, Lim SN, Su MY, Yeh CT. Dynamic bioenergetic alterations in colorectal adenomatous polyps and adenocarcinomas. EBioMedicine (2019) 44:334-45. doi: 10.1016/j.ebiom.2019.05.031

16. Bu P, Chen KY, Xiang K, Johnson C, Crown SB, Rakhilin N, et al. Aldolase BMediated Fructose Metabolism Drives Metabolic Reprogramming of Colon Cancer Liver Metastasis. Cell Metab (2018) 27:1249. doi: 10.1016/j.cmet.2018.04.03

17. Melo SA, Luecke LB, Kahlert C, Fernandez AF, Gammon ST, Kaye J, et al. Glypican-1 identifies cancer exosomes and detects early pancreatic cancer. Nature (2015) 523(7559):177-82. doi: 10.1038/nature14581

18. Chamorro-Jorganes A, Araldi E, Rotllan N, Cirera-Salinas D, Suarez Y. Autoregulation of glypican-1 by intronic microRNA-149 fine tunes the angiogenic response to FGF2 in human endothelial cells. J Cell Ence (2014) 127(6):1169. doi: $10.1242 /$ jcs. 130518

19. Pamidimukkala NV, Leonard MK, Snyder D, McCorkle JR, Kaetzel DM. Metastasis Suppressor NME1 Directly Activates Transcription of the ALDOC Gene in Melanoma Cells. Anticancer Res (2018) 38(11):6059-68. doi: 10.21873/anticanres.12956

20. Debangshu S, Semenza GL. Metabolic adaptation of cancer and immune cells mediated by hypoxia-inducible factors. Biochim Biophys Acta (BBA) Rev Cancer (2018) 1870:15-22. doi: 10.1016/j.bbcan.2018.07.002

21. Brooks JM, Menezes AN, Ibrahim M, Archer L, Lal N, Bagnall CJ, et al. Development and validation of a combined hypoxia and immune prognostic classifier for head and neck cancer. Clin Cancer Res (2019) 17):5315-28. doi: 10.1158/1078-0432.CCR-18-3314

22. Yang L, Roberts D, Takhar M, Erho N, Bibby BAS, Thiruthaneeswaran N, et al. Development and Validation of a 28-gene Hypoxia-related Prognostic Signature for Localized Prostate Cancer. EBioMedicine (2018) 31:182-9. doi: 10.1016/j.ebiom.2018.04.019

23. Zhou D, Huang C, Lin Z. Macrophage polarization and function with emphasis on the evolving roles of coordinated regulation of cellular signaling pathways. Cell SIGNALLING (2014) (2):192-7. doi: 10.1016/j.cellsig.2013.11.004

24. Sethi N, Kikuchi O, Mcfarland J, Zhang Y, Bass AJ. Mutant p53 induces a hypoxia transcriptional program in gastric and esophageal adenocarcinoma. JCI Insight (2019) 4(15):e128439. doi: 10.1172/jci.insight.128439

25. Hao N-B, Lü M-H, Fan Y-H, Cao Y-L, Zhang Z-R, Yang S-M, et al. Macrophages in tumor microenvironments and the progression of tumors. Clin Dev Immunol (2012) 2012:948098. doi: 10.1155/2012/948098

26. Murray PJ. Macrophage Polarization. Annu Rev Physiol (2016) 79(1):541. doi: 10.1146/annurev-physiol-022516-034339

27. Sun J, Zhang Y, Yang M, Yang M, Zhang Y, Xie Q, et al. Hypoxia induces Tcell apoptosis by inhibiting chemokine $\mathrm{C}$ receptor 7 expression: the role of adenosine receptor A2. Cell Mol Immunol (2010) 7(1):77-82. doi: 10.1038/ cmi.2009.105

28. Urata S, Izumi K, Hiratsuka K, Maolake A, Natsagdorj A, Shigehara K, et al. $\mathrm{C}-\mathrm{C}$ motif ligand 5 promotes migration of prostate cancer cells in the prostate 
cancer bone metastasis microenvironment. Cancer Ence (2018) 109(3):72431. doi: $10.1111 /$ cas.13494

29. Izumi K, Fang L, Mizokami A, Namiki M, Li L, Lin W, et al. Targeting the androgen receptor with siRNA promotes prostate cancer metastasis through enhanced macrophage recruitment via CCL2/CCR2-induced STAT3 activation. EMBO Mol Med (2013) 5(9):1383-401. doi: 10.1002/emmm.201202367

30. Hu G, Tzeng H, Chen P, Wang C, Zhao Y, Wang Y, et al. Correlation between CCL4 gene polymorphisms and clinical aspects of breast cancer. Int J Of Med Sci (2018) 11):1179-86. doi: 10.7150/ijms.26771

31. Lin W, Xu D, Austin CD, Caplazi P, Senger K, Sun Y, et al. Function of CSF1 and IL34 in Macrophage Homeostasis, Inflammation, and Cancer. Front Immunol (2019) 10:2019. doi: 10.3389/fimmu.2019.02019

32. Baghdadi M, Endo H, Takano A, Ishikawa K, Kameda Y, Miyagi Y, et al. High co-expression of IL-34 and M-CSF correlates with tumor progression and poor survival in lung cancers. Sci Rep (2018) 8. doi: 10.1038/s41598-017-18796-8

33. Barreda DR, Hanington PC, Belosevic M. Regulation of myeloid development and function by colony stimulating factors. Dev Comp Immunol (2004) 28:509-54. doi: 10.1016/j.dci.2003.09.010
34. Olingy CE, Dinh HQ, Hedrick CC. Monocyte Heterogeneity and Functions in Cancer. J Leukocyte Biol (2019) 106(2):309-22. doi: 10.1002/JLB.4RI0818311R

35. Poh AR, Matthias E. Targeting Macrophages in Cancer: From Bench to Bedside. Front Oncol (2018) 8:49. doi: 10.3389/fonc.2018.00049

Conflict of Interest: The authors declare that the research was conducted in the absence of any commercial or financial relationships that could be construed as a potential conflict of interest.

Copyright (c) 2021 Zhang, Wang, Wang, Zhao, Zhang, Zhang and Xu. This is an open-access article distributed under the terms of the Creative Commons Attribution License (CC BY). The use, distribution or reproduction in other forums is permitted, provided the original author(s) and the copyright owner(s) are credited and that the original publication in this journal is cited, in accordance with accepted academic practice. No use, distribution or reproduction is permitted which does not comply with these terms. 